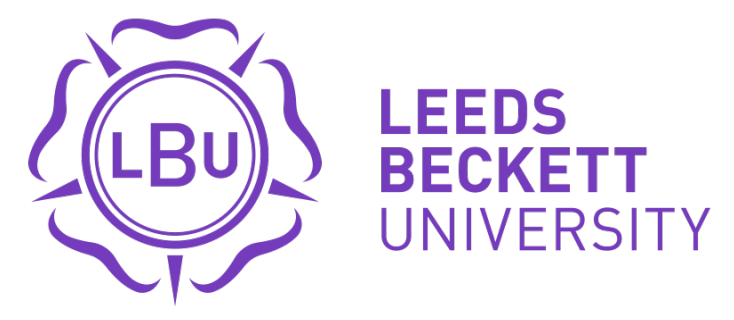

Citation:

Hobkirk, JP and King, RF and Gately, P and Pemberton, P and Smith, A and Barth, JH and Harman, N and Davies, I and Carroll, S (2013) The predictive ability of triglycerides and waist (hypertriglyceridemic waist) in assessing metabolic triad change in obese children and adolescents. Metabolic syndrome and related disorders, 11 (5). 336 - 342. ISSN 1540-4196 DOI: https://doi.org/10.1089/met.2012.0152

Link to Leeds Beckett Repository record:

https://eprints.leedsbeckett.ac.uk/id/eprint/218/

Document Version:

Article (Accepted Version)

The aim of the Leeds Beckett Repository is to provide open access to our research, as required by funder policies and permitted by publishers and copyright law.

The Leeds Beckett repository holds a wide range of publications, each of which has been checked for copyright and the relevant embargo period has been applied by the Research Services team.

We operate on a standard take-down policy. If you are the author or publisher of an output and you would like it removed from the repository, please contact us and we will investigate on a case-by-case basis.

Each thesis in the repository has been cleared where necessary by the author for third party copyright. If you would like a thesis to be removed from the repository or believe there is an issue with copyright, please contact us on openaccess@leedsbeckett.ac.uk and we will investigate on a case-by-case basis. 


\title{
The Predictive Ability of Triglycerides and Waist (Hypertriglyceridemic Waist) in Assessing Metabolic Triad Change in Obese Children and Adolescents
}

\author{
James P. Hobkirk, PhD, ${ }^{1}$ Roderick F. King, PhD, ${ }^{2}$ Paul Gately, PhD, ${ }^{2}$ Philip Pemberton, MSc, ${ }^{3}$ \\ Alexander Smith, $\mathrm{PhD},{ }^{3}$ Julian $\mathrm{H}$. Barth, MD, Nicola Harman, $\mathrm{PhD}^{5}$ \\ Ian Davies, $\mathrm{PhD},{ }^{6}$ and Sean Carroll, $\mathrm{PhD}^{7}$
}

\begin{abstract}
Background: The metabolic triad [fasting insulin, apolipoprotein B, and low-density lipoporotein (LDL) peak particle density] is characteristic of increased intra-abdominal adipose tissue and insulin resistance and can be predicted by the simple and adoptable screening tool, the hypertriglyceridemic waist. The associations between hypertriglyceridemic waist components [fasting triglycerides (TG) and waist circumference cut-points derived from a child-specific metabolic syndrome definition] with the metabolic triad were examined in obese youth before and after weight loss.

Methods: A continuous metabolic triad score (MTS) was calculated as a cumulative and standardized residual score of fasting insulin, apolipoprotein B, and LDL peak particle density ( $z$-scores of the metabolic triad variables regressed onto age and sex). The predictive ability of TG and waist in assessing metabolic triad change was undertaken in 75 clinically obese boys and girls, aged $8-18$, body mass index (BMI) $34.2 \pm 6.4 \mathrm{~kg} / \mathrm{m}^{2}$ before and after weight loss.

Results: Fasting TG concentrations $\left(r^{2}=0.216, P<0.0001\right)$ and waist circumference $\left(r^{2}=0.049, P=0.019\right)$ were both significant independent predictors of the cumulative MTS, together accounting for $26.5 \%$ of its total variance. All cardiometabolic risk factors [except a reduction in high-density lipoprotein cholesterol (HDL-C)] were favorably modified following weight loss. Fasting TG change was the only significant predictor of the MTS change $\left(r^{2}=0.177, P<0.0001\right)$. Waist circumference was not a significant predictor of MTS change.

Conclusion: The reduction in fasting TG concentration (but not waist circumference) was the only significant predictor of MTS change. Fasting TG may be the most important metabolic syndrome component to best characterize the metabolic heterogeneity in obese cohorts and the changes in metabolic risk in clinically obese youth.
\end{abstract}

\section{Introduction}

ThE various combInations of metabolic syndrome 1 components may differ in clinical significance. ${ }^{1}$ In obese youth, waist circumference has limited discriminatory potential to identify those with high or low levels of intra-abdominal adipose tissue (IAAT), ${ }^{2}$ and most obese children remain normoglycemic despite underlying insulin resistance measured by clamps. ${ }^{3}$ Therefore, the sensitivity of waist circumference and or glucose to characterize increased cardiometabolic risk may be limited. The constellation of hyperinsulinemia, high apolipoprotein $B(A p o B)$, and a small low-density lipoprotein (LDL) particle size, termed the "metabolic triad" was associated with a substantially

${ }^{1}$ Department of Academic Endocrinology, Diabetes and Metabolism, Hull York Medical School, University of Hull, Hull, United Kingdom.

${ }^{2}$ Carnegie Research Institute, Leeds Metropolitan University, Leeds, United Kingdom.

${ }^{3}$ Specialist Assay Laboratory, Clinical Biochemistry, Manchester Royal Infirmary, Manchester, United Kingdom.

${ }^{4}$ Clinical Biochemistry, Leeds General Infirmary, Leeds, United Kingdom.

${ }^{5}$ Clinical Trials Research Centre, University of Liverpool, Liverpool, United Kingdom.

${ }^{6}$ Faculty of Education, Community and Leisure, Liverpool John Moores University, Liverpool, United Kingdom.

${ }^{7}$ Department of Sport, Health and Exercise Science, University of Hull, Hull, United Kingdom. 
increased risk of cardiovascular disease (CVD) in middleaged men. ${ }^{4}$ The metabolic triad was characteristic of middleaged men with high levels of IAAT and insulin resistance. The increased CVD risk associated with the metabolic triad was independent of LDL-cholesterol (LDL-C) and high density lipoprotein-cholesterol (HDL-C) concentration. ${ }^{4}$ However, the metabolic triad components are rarely measured in clinical settings due to accessibility, cost, and standardization issues. The hypertriglyceridemic waist phenotype (HyperTG waist) is a simple screening tool, using the combination of waist circumference and fasting triglycerides (TG). The HyperTG waist has previously been shown to predict the metabolic triad with high $(>80 \%)$ sensitivity and specificity in middle-aged adults. ${ }^{5}$ However, the HyperTG waist and the metabolic triad have not been previously investigated in obese youth, especially in response to weight loss. The aim of the present study was to evaluate the relative importance of waist circumference or fasting TG changes on the continuous metabolic triad score (MTS) change. Furthermore, we assessed whether glucose (as a surrogate for insulin resistance) or systolic blood pressure (SBP) accounted for any of the variance in MTS change.

\section{Methods}

Participants were attendees of the Carnegie International Camp (CIC), a multifactorial residential weight loss program. Details of the program are discussed elsewhere. ${ }^{6}$ We recruited both children $(n=15)$ and adolescents $(n=60)$ aged between 8 and 18 years who attended from the United Kingdom and Europe. Recruitment was based on self or parental application, medical recommendation, or social service referral between the years of 2004 and 2006. Acceptance into the program was reliant on having a body mass index (BMI) above the age-related cutoffs for overweight as suggested by Cole. ${ }^{7}$ There were no other specific inclusion criteria, which resulted in a group of heterogeneous children and adolescents with respect to age, sexual maturation, and degree of obesity. The subjects were primarily caucasian ( $n=72$, Asian $n=1$ and black $n=2$ ). Tanner stages, family history of obesity, type 2 diabetes (T2D), or CVD were not collected.

\section{Ethical approval}

Ethical approval was granted by Leeds West National Health Service Research Ethics Committee, Leeds, United Kingdom. All participants and parents provided written informed consent prior to inclusion in the study. More specifically, all participants and parents provided written consent and verbal assent for blood withdrawal. However, our sample size was affected by the inability to obtain venous access at baseline and withdrawal of consent for postintervention blood withdrawal.

The program incorporated energy restriction, physical activity/exercise, and lifestyle education. All children and adolescents undertook a daily schedule of physical activity that was a standardized and compulsory component of the weight loss program, consisting of six 1-hr sessions each day. We did not monitor directly the intensity throughout the sessions via heart rate monitors nor did we objectively calculate the amount of physical activity by accelerometry.

Energy intake (kcal $\cdot$ day $\left.^{-1}\right)$ was provided as three meals and a snack each day and was based on an approximation of basal metabolic rate (BMR), using the equations of Schofield using age and body mass. ${ }^{8}$ Each participant was assigned to one of four specific dietary groups ranging from 1050 to 3050 $\mathrm{kcal} \cdot \mathrm{day}^{-1}$, based upon their age and body mass. The daily energy intake contained $15 \%$ protein, $30 \%-35 \%$ fat, and $50 \%-55 \%$ carbohydrate. Due to the residential setting, adherence to the program could be ascertained. Plate waste was also measured randomly for all meals once a week in a subsample of children. ${ }^{9}$

Each participant also engaged in four 1-hr educational sessions per week covering food choices, the balance of good health, portion control, and stimulus control. Sessions developed skills regarding behavior change, cognitive reinforcement, goal setting, problem solving, acquiring social support, and issues regarding bullying. ${ }^{10}$

\section{Anthropometric and blood pressure measurements}

Waist circumference was assessed at the iliac crest, as recommended by the National Cholesterol Education Program Adult Treatment Panel III (NCEP ATP III) to the nearest $0.1 \mathrm{~cm}$ using a standard clinical tape measure. Bioelectrical impedance analysis (BIA) measures were made using a Tanita TBF-310 (Tanita Corp, Tokyo, Japan). BIA estimated total body water and calculated fat mass was obtained using equations derived by Jebb et al. ${ }^{11}$

Blood pressure was measured using a mercury sphygmomanometer (Mercurial BK1001) on the left arm. All subjects sat quietly for $5 \mathrm{~min}$ prior to measurement. A single measurement was taken in the semisupine position. In a subsample of participants (2006 cohort), a digital blood pressure monitoring device as opposed to manual method was used (Omron HEM-773AC, UK). Measurement protocols defined the appropriate cuff size to be used in all measurements.

\section{Biochemical measurements}

Fasting blood samples (12-13.5 hr) were drawn by venipuncture. Bloods were stored on ice and analyzed within $3 \mathrm{hr}$ of venipuncture. Analyses for lipids, glucose, and insulin were undertaken at the Department of Clinical Biochemistry, Leeds General Infirmary, UK, using routine clinical analyses. Insulin was analyzed by an ADVIA chemiluminescent sandwich immunoassay using a monoclonal mouse anti insulin antibody. The intra-assay coefficient of variation for insulin was $3.5 \%$.

\section{Apolipoprotein B}

ApoB was analyzed at the specialist Assay Laboratory, Department of Clinical Sciences, Manchester Royal Infirmary after storage at $-80^{\circ} \mathrm{C}$, by a sandwich enzyme-linked immunosorbent assay (ELISA) technique employing a goat anti-human ApoB antibody (Abcam ab7616, Cambridge) and horseradish peroxidase (HRP)-labeled goat anti-human ApoB (Abcam ab20047). Following incubation, color development was measured at $490 \mathrm{~nm}$ on a Dynatech MR 7000 plate reader. The within-batch coefficient of variation was $8.7 \%$ and the between-batch coefficient of variation was $9.9 \%$.

\section{LDL particle density}

LDL peak particle density was measured in the Carnegie Research Institute, Leeds Metropolitan University, by iodixanol 
gradient ultracentrifugation and digital photography, after storage at $-80^{\circ} \mathrm{C}$. Briefly, $1.52 \mathrm{~mL}$ of plasma was added to $0.4 \mathrm{~mL}$ of $60 \% \mathrm{wt} / \mathrm{vol}$ iodixanol (Axis Shield, UK) and stained by addition of diluted Coomassie Blue $(50 \mathrm{mg} / \mathrm{mL}$ phosphatebuffered saline). This was layered under $3.4 \mathrm{~mL}$ of iodixanol $9 \%$ (wt/vol) in Beckman Optiseal ${ }^{\mathrm{TM}}$ tubes. Tubes were placed in a Beckman NVT 65.2 rotor and centrifuged at $65,000 \mathrm{rpm}$ for $2.5 \mathrm{hr}$. A Nikon digital camera D-100 was used to photograph the tubes, and these images were downloaded into gel scan software (Total Lab, Non Linear Dynamics, UK). A migration distance $\left(R^{\mathfrak{f}}\right)$ corresponded to a specific density based on prior calibration experiments. It is also important to note that LDL density in iodixanol is lower than using salt density gradient ultracentrifugation due to the iso-osmotic properties of iodixanol. This method has been validated against both salt density gradient ultracentrifugation and gradient gel electrophoresis. ${ }^{12}$

Reproducibility for LDL peak particle density was assessed in $(n=11)$ in healthy volunteers. The coefficient of variation was $0.1 \%$ when samples were measured fresh, at 1 week, 1 month, and 6 months (unpublished data).

\section{HyperTG waist phenotype definition using existing pediatric metabolic syndrome cut points}

The HyperTG metabolic screening tool was derived from a child-specific metabolic syndrome definition. ${ }^{13}$ For waist circumference cut points, deFerranti et al. ${ }^{13}$ (2004) used the $70^{\text {th }}$ percentile for adult men described by Zhu et al. ${ }^{14}$ The TG cut points of deFerranti et al. corresponded to $1.1 \mathrm{mmol} /$ L. The HyperTG waist phenotype was considered present when an individual exceeded both the waist circumference and fasting TG cut points of the metabolic syndrome definition. No reference values or cutoffs are available for any components of the metabolic triad in children or adolescents. Accordingly, we used a cumulative residual score of fasting insulin, ApoB, and LDL peak particle density (after regression on to age and sex) to generate a continuous MTS. This is supported by Lamarche, ${ }^{4}$ who found that the metabolic triad variables were more frequently observed in combination than in isolation. A higher MTS infers a higher cumulative burden of the three MTS components. A continuous scoring approach may also overcome the problems of dichotomous cut points in risk prediction. ${ }^{15}$

\section{Statistics}

All variables were examined for normality of distribution. Fasting TG and insulin concentrations were skewed and were logarithmically $(\mathrm{Ln})$ transformed prior to analysis. The HyperTG waist phenotype was calculated as the number of participants who exceeded both the waist and TG metabolic syndrome cut points. The MTS was calculated by transforming all metabolic triad variables (insulin, $A p o B$, and LDL peak particle density) to standardized residual scores after transforming to $z$-scores and then regressing onto age and sex. The MTS was calculated as the sum of the cumulative standardized residual scores. It was not possible to have a dichotomous metabolic triad positive/negative diagnosis, because no cutoffs for these metabolic variables exist in children and adolescents. Independent $t$-tests were used to examine differences in anthropometric and cardiometabolic risk factors between participants with and without the HyperTG waist phenotype. The differences in MTS between those who were positive and negative for HyperTG waist components were established by one-way analysis of variance (ANOVA). Paired $t$-tests were undertaken to assess changes in anthropometric and cardiometabolic variables after acute weight loss. The change in the MTS was calculated by subtracting the post-MTS score from the pre-MTS for each participant.

Multiple regression analysis was conducted to assess the contributions of waist circumference, Ln TG, SBP, and glucose change to predict the baseline MTS and changes in the MTS. All analyses were performed on SPSS version 14.0 and statistical significance was assumed at $P<0.05$.

\section{Results}

The prevalence of the HyperTG waist according to deFerranti ${ }^{3}$ was $40 \%$. Several mean differences in metabolic variables were evident using the cut points of deFerranti. ${ }^{13}$ LDL density; ApoB was significantly higher in the HyperTG waist group, but insulin concentrations were not significantly different $P=0.09$ (Table 1 ). There was no significant association of waist circumference with the MTS. In contrast; fasting TG concentration was significantly associated with the cumulative MTS. Amongst participants with raised fasting TG (deFerranti fasting TG $>1.10 \mathrm{mmol} / \mathrm{L}$ ), the MTS was $+0.82 \pm 1.95$ compared to $-0.57 \pm 1.54$ in those with TG concentrations below the cut point $P<0.0001$.

Multiple stepwise regression analyses were undertaken to establish whether each HyperTG waist component (waist

Table 1. ANTHRopometric, Body Composition, and Cardiometabolic Variables According to THE HyperTG Phenotype Based on Waist and TGs Cut Points Derived from a Metabolic Syndrome Definition (DeFerRanti et AL. 2004 $4^{13}$ )

\begin{tabular}{|c|c|c|c|}
\hline & \multicolumn{3}{|c|}{ deFerranti et al. (2004) } \\
\hline & $\begin{array}{c}\text { HyperTG } \\
\text { negative } \\
\mathrm{n}=45(12 \\
\text { boys } / 33 \text { girls })\end{array}$ & $\begin{array}{c}\text { HyperTG } \\
\text { positive } \\
\mathrm{n}=30(15 \\
\text { boys/15 girls) }\end{array}$ & $\mathrm{P}$ value \\
\hline Body mass $(\mathrm{kg})$ & $94.7 \pm 23.3$ & $93.6 \pm 20.7$ & 0.83 \\
\hline $\mathrm{BMI}\left(\mathrm{kg} / \mathrm{m}^{2}\right)$ & $34.2 \pm 6.2$ & $34.1 \pm 6.6$ & 0.97 \\
\hline SDs BMI & $3.03 \pm 0.63$ & $3.12 \pm 0.54$ & 0.53 \\
\hline Waist $(\mathrm{cm})^{\mathrm{a}}$ & $111.2 \pm 13.2$ & $110.8 \pm 13.6$ & 0.89 \\
\hline Fat mass $(\mathrm{kg})$ & $39.2 \pm 14.5$ & $39.0 \pm 12.8$ & 0.94 \\
\hline $\mathrm{TC}\left(\mathrm{mmol} \cdot \mathrm{L}^{-1}\right)$ & $4.02 \pm 0.70$ & $4.34 \pm 0.64$ & $<0.05$ \\
\hline $\mathrm{LDL}-\mathrm{C}\left(\mathrm{mmol} \cdot \mathrm{L}^{-1}\right)$ & $2.43 \pm 0.61$ & $2.54 \pm 0.52$ & 0.42 \\
\hline $\mathrm{TG}\left(\mathrm{mmol} \cdot \mathrm{L}^{-1}\right)$ & $0.79 \pm 0.15$ & $1.58 \pm 0.43$ & $<0.001$ \\
\hline $\mathrm{HDL}-\mathrm{C}\left(\mathrm{mmol} \cdot \mathrm{L}^{-1}\right)$ & $1.25 \pm 0.20$ & $1.12 \pm 0.28$ & $<0.05$ \\
\hline LDL density $\left(\mathrm{g} \cdot \mathrm{mL}^{-1}\right)$ & $1.024 \pm 0.002$ & $1.026 \pm 0.003$ & $<0.01$ \\
\hline $\mathrm{ApoB}\left(\mathrm{mg} \cdot \mathrm{dL}^{-1}\right)$ & $85.1 \pm 21.7$ & $96.9 \pm 21.2$ & $<0.05$ \\
\hline Glucose $\left(\mathrm{mmol} \cdot \mathrm{L}^{-1}\right)$ & $4.76 \pm 0.44$ & $4.83 \pm 0.33$ & 0.44 \\
\hline Insulin $\left(\mathrm{mU} \cdot \mathrm{L}^{-1}\right)$ & $15.9 \pm 11.0$ & $20.3 \pm 10.8$ & 0.09 \\
\hline $\mathrm{SBP}(\mathrm{mmHg})$ & $120 \pm 15$ & $120 \pm 12$ & 0.88 \\
\hline $\mathrm{DBP}(\mathrm{mmHg})$ & $72 \pm 11$ & $72 \pm 10$ & 0.72 \\
\hline MTS & $-0.57 \pm 1.54$ & $0.82 \pm 1.95$ & $<0.01$ \\
\hline
\end{tabular}

${ }^{\mathrm{a}}$ Iliac crest (deFerranti et al. $2004^{13}$ ).

HyperTG, hypertriglyceridemic; TG, triglycerides; BMI, body mass index; SD, standard deviation; TC, total cholesterol; LDL-C, low-density lipoprotein cholesterol; HDL-C, high-density lipoprotein cholesterol; ApoB, apolipoprotein B; SBP, systolic blood pressure; DBP, diastolic blood pressure; MTS, metabolic triad score. 
Table 2. Anthropometric, Body Composition, and Cardiometabolic Risk Factor Changes AFTER ACUTE WeIght Loss IN Boys AND GirLs

\begin{tabular}{|c|c|c|c|c|c|}
\hline & \multicolumn{2}{|c|}{ Pre Mean $\pm S D$} & \multicolumn{2}{|c|}{ Post Mean $\pm S D$} & \multirow{2}{*}{$\begin{array}{c}\text { P values } \\
\text { (between sex) }\end{array}$} \\
\hline & Boys & Girls & Boys & Girls & \\
\hline Body mass (kg) & $99.3 \pm 26.5$ & $91.4 \pm 19.0$ & $93.4 \pm 23.8$ & $86.3 \pm 18.4$ & 0.32 \\
\hline $\mathrm{BMI}\left(\mathrm{kg} \cdot \mathrm{m}^{-2}\right)$ & $33.6 \pm 6.5$ & $34.5 \pm 6.4$ & $31.6 \pm 5.6$ & $32.5 \pm 6.2$ & 0.94 \\
\hline SDs BMI & $3.09 \pm 0.60$ & $3.05 \pm 0.59$ & $2.88 \pm 0.61$ & $2.79 \pm 0.65$ & 0.07 \\
\hline Waist iliac crest $(\mathrm{cm})$ & $111.8 \pm 15.1$ & $110.8 \pm 12.0$ & $106.3 \pm 12.7$ & $107.3 \pm 13.1$ & 0.30 \\
\hline Fat mass $(\mathrm{kg})$ & $35.9 \pm 14.9$ & $40.7 \pm 12.4$ & $29.0 \pm 11.5$ & $36.1 \pm 11.9$ & 0.01 \\
\hline SBP (mmHg) & $125 \pm 16$ & $117 \pm 10$ & $119 \pm 11$ & $115 \pm 11$ & 0.20 \\
\hline DBP (mmHg) & $74 \pm 14$ & $70 \pm 8$ & $66 \pm 9$ & $69 \pm 11$ & 0.01 \\
\hline $\mathrm{TC}\left(\mathrm{mmol} \cdot \mathrm{L}^{-1}\right)$ & $4.17 \pm 0.62$ & $4.13 \pm 0.73$ & $3.15 \pm 0.42$ & $3.33 \pm 0.64$ & 0.05 \\
\hline $\mathrm{LDL}-\mathrm{C}\left(\mathrm{mmol} \cdot \mathrm{L}^{-1}\right)$ & $2.47 \pm 0.55$ & $2.47 \pm 0.59$ & $1.72 \pm 0.37$ & $1.84 \pm 0.49$ & 0.15 \\
\hline $\mathrm{HDL}-\mathrm{C}\left(\mathrm{mmol} \cdot \mathrm{L}^{-1}\right)$ & $1.15 \pm 0.22$ & $1.23 \pm 0.25$ & $1.07 \pm 0.22$ & $1.13 \pm 0.24$ & 0.6 \\
\hline $\mathrm{TG}\left(\mathrm{mmol} \cdot \mathrm{L}^{-1}\right)$ & $1.25 \pm 0.61$ & $1.03 \pm 0.39$ & $0.86 \pm 0.31$ & $0.83 \pm 0.31$ & 0.05 \\
\hline LDL density $\left(\mathrm{g} \cdot \mathrm{mL}^{-1}\right)$ & $1.026 \pm 0.003$ & $1.024 \pm 0.002$ & $1.023 \pm 0.001$ & $1.023 \pm 0.002$ & 0.01 \\
\hline LDL pattern B $(\%)$ & $38.9 \pm 15.3$ & $30.2 \pm 7.7$ & $32.9 \pm 10.7$ & $30.2 \pm 7.7$ & 0.01 \\
\hline $\mathrm{ApoB}\left(\mathrm{mg} \cdot \mathrm{dL}^{-1}\right)$ & $86.9 \pm 21.3$ & $90.6 \pm 22.2$ & $66.8 \pm 13.5$ & $70.7 \pm 18.4$ & 0.11 \\
\hline Leptin (ng $\cdot \mathrm{mL}^{-1}$ ) & $87.4 \pm 39.6$ & $148.1 \pm 72.9$ & $31.1 \pm 19.5$ & $70.9 \pm 55.9$ & 0.96 \\
\hline Glucose $\left(\mathrm{mmol} \cdot \mathrm{L}^{-1}\right)$ & $4.85 \pm 0.40$ & $4.76 \pm 0.40$ & $4.64 \pm 0.22$ & $4.51 \pm 0.35$ & 0.18 \\
\hline Insulin $\left(\mathrm{mU} \cdot \mathrm{L}^{-1}\right)$ & $20.7 \pm 13.7$ & $15.7 \pm 9.0$ & $14.4 \pm 10.4$ & $11.6 \pm 5.5$ & 0.29 \\
\hline HOMA-IR & $4.4 \pm 3.2$ & $3.4 \pm 1.9$ & $3.0 \pm 2.3$ & $2.3 \pm 1.1$ & 0.43 \\
\hline
\end{tabular}

SD standard deviation; BMI, body mass index; SBP, systolic blood pressure; DBP, diastolic blood pressure; TC, total cholesterol; LDL-C low-density lipoprotein cholesterol; HDL-C, high-density lipoprotein; TG, triglycerides; ApoB, apolipoprotein B; HOMA-IR, homeostasis model assessment of insulin resistance.

circumference and fasting TG concentration) predicted the cumulative MTS at pre intervention (after regressing onto age and sex). Ln TG concentration $(P<0.0001)$, and waist circumference $(P=0.019)$ were both independent predictors of the MTS, together, accounting for $26.5 \%$ of the total variance. Ln TG accounted for the majority $\left(r^{2}=0.216\right.$ or $\left.21.6 \%\right)$ of the variance in the cumulative MTS at pre intervention. Table 2 shows highly significant improvements in all cardiometabolic risk factors (except HDL-C) following weight loss. Waist circumference decreased by $-5.5 \pm 6.0 \mathrm{~cm}$ in boys and by $-3.5 \pm 4.5$ in girls. TG decreased by $-0.39 \pm 0.53$ in boys and by $-0.20 \pm 0.33$ in girls. The prevalence of the HyperTG phenotype decreased from $40 \%$ to $0 \%$ deFerranti. ${ }^{13}$ Multiple regression analysis was undertaken to examine changes in waist circumference, LnTG, glucose and SBP with change in the MTS after acute weight loss. The model accounted for $19.6 \%$ of the variance in the MTS (Table 3 ). In stepwise multiple regressions Ln TG change was the only predictor of MTS change $\left(r^{2}=0.177, P<0.0001\right)$. The change in waist, SBP, or glucose was not an independent predictor

Table 3. Summary of Multiple Regression Model. FOR THE ACCOUNTED VARIANCE IN THE MTS CHANGE IN BOTH Boys AND GIRLS COMBINED

\begin{tabular}{lcccc}
\hline & $\mathrm{R}^{2}$ & Adjusted $\mathrm{R}^{2}$ & Beta & $\mathrm{P}$ \\
\hline Ln TG & 0.243 & 0.196 & 0.408 & $P<0.0001$ \\
Waist & & & 0.095 & 0.39 \\
SBP & & & 0.117 & 0.29 \\
Glucose & & & -0.175 & 0.11 \\
\hline
\end{tabular}

${ }^{a}$ On stepwise multiple regression analysis $\mathrm{Ln}$ TG accounted for $17.7 \%$ of the variance of MTS change.

MTS, metabolic triad score; Ln TG, triglycerides logarithm; SBP, systolic blood pressure. of MTS change. We also established sexual dimorphism in these relationships by conducting multiple regressions in boys and girls separately, including waist, Ln TG, glucose and SBP as dependent variables. Waist circumference, SBP, and glucose were omitted from both the models. In contrast, Ln TG accounted for the majority of the variance in the MTS change in boys $(25.1 \%) r^{2}=0.251$. However, in the girls Ln TG accounted for $(9.6 \%) r^{2}=0.096$ of the variance in the MTS change. The relationship between sex and changes in Ln TG and the MTS are shown in Fig. 1A (boys) and 1B (girls).

\section{Discussion}

We have shown for the first time the utility of fasting TG to predict the metabolic triad (a constellation of CVD risk factors associated with insulin resistance, IAAT, and metabolic obesity) in both a cross-sectional study and in response to highly significant acute weight loss in clinically obese youth. This is the first study that has simultaneously modified the components of the metabolic triad in response to weight loss in clinically obese youth, although some randomized clinical weight loss trials do show a lowering of traditional cardiometabolic risk factors. ${ }^{16,17}$ Multiple regression analysis including waist, Ln TG, SBP, and glucose in the model showed that $L n$ TG reduction was the only significant independent predictor of MTS change (Table 3).

Lemieux ${ }^{5}$ showed that in middle-aged men fasting TG was most strongly associated with the metabolic triad. In this study, CVD risk increased between categories of TG $(<2.0$ $\mathrm{mmol} / \mathrm{L}$ vs. $>2.0 \mathrm{mmol} / \mathrm{L})$, whereas these relationships were not evident for waist circumference $(<90.0 \mathrm{~cm}$ vs. $>90.0 \mathrm{~cm}$ ) and the prevalence of the metabolic triad increased from $12 \%$ in those with waist circumference between $90 \mathrm{~cm}$ and $100 \mathrm{~cm}$ but a TG level below $2.0 \mathrm{mmol} / \mathrm{L}$ to $83 \%$ in those within the same waist category but with a TG level above 

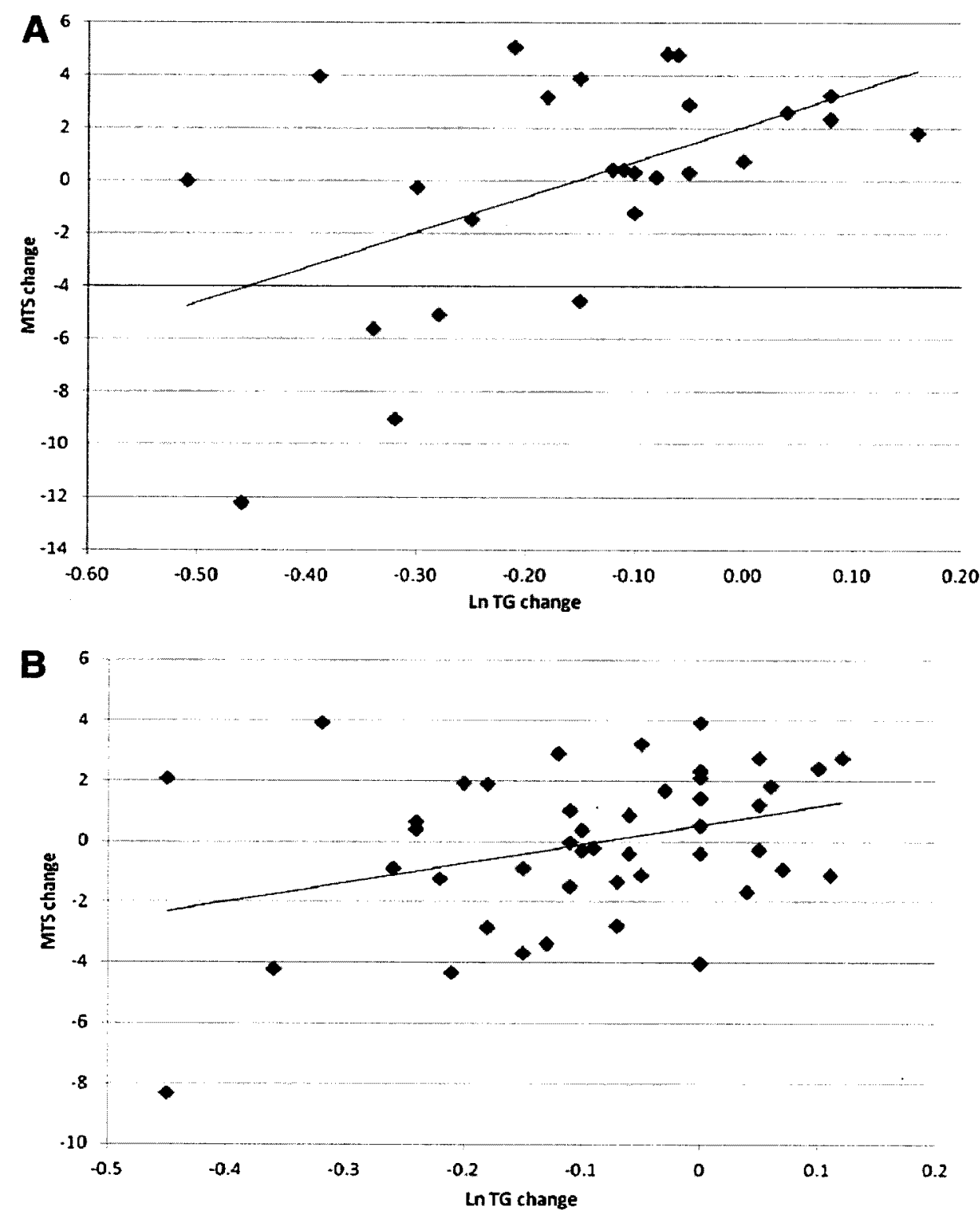

FIG. 1. The relationship between triglycerides logarithm (Ln TG) and the metabolic triad score (MTS) (after regressing on to age and sex) in boys (A) and girls (B).

$2.0 \mathrm{mmol} / \mathrm{L}$. The increased emphasis on TG within the metabolic syndrome framework is strengthened by novel approaches to elucidate the underlying pathophysiology of cardiometabolic risk and the recent literature emphasizing the importance of TG as a biomarker of cardiometabolic risk. ${ }^{3,18,19}$

In a study of 118 severely obese adolescents (BMI range $35.5-39.1 \mathrm{~kg} / \mathrm{m}^{2}$ ), waist circumference and fasting glucose values were very similar across tertiles of IAAT (as measured by magnetic resonance imaging), whereas the median TG showed a stepwise increase across tertiles of IAAT. ${ }^{2}$ In another study of severely obese adolescents (BMI 35.7 \pm 0.9 ), MRI-derived subcutaneous abdominal adipose tissue (SAAT) and glucose were no different in those with insulin resistance (IR). In contrast, those with higher TG $(1.62 \pm 0.02$ $\mathrm{mmol} / \mathrm{L}$ ) had higher IR, fasting insulin, hepatic fat, IAAT, intramyocellular lipid, and small dense LDL particles. ${ }^{20}$

There is also considerable epidemiological evidence to support the use of TG and the TG/HDL ratio to identify insulin resistance in purely obese cohorts of varying ages and sexes. This is particularly useful when considering the metabolic heterogeneity within obese cohorts, notably when these individuals remain normoglycemic and exceed all recognized waist cutoffs. McLaughlin et al. ${ }^{21}$ evaluated the ability of BMI (waist circumference not measured), fasting glucose, and TG concentrations to identify subjects with clamp-derived insulin resistance. It was shown that TG was the most sensitive to identify insulin-resistant overweight adults. ${ }^{21} \mathrm{~A}$ similar study in obese adolescents showed that only fasting TG cut point of $1.20 \mathrm{mmol} / \mathrm{L}$ had a sensitivity of $70 \%$ and specificity of $77 \%$ 
for identifying obese children in the lowest tertile of insulin sensitivity, measured by clamps. ${ }^{22}$ Furthermore, waist circumference and fasting insulin (glucose not measured) were not significantly different between those stratified above and below the $1.2 \mathrm{mmol} / \mathrm{L}(106 \mathrm{~mL} / \mathrm{dL})$ arbitrary cutoff.

In one study, the TG-to-HDL ratio was significantly associated with Framingham risk (22.7\% of the variance) and was more powerful than sex, blood pressure, waist-to-hip ratio, non-HDL, and the composite metabolic syndrome. ${ }^{23}$ In a prospective study, the TG-to-HDL ratio and the composite metabolic syndrome diagnosis were the strongest predictors of the development of IFG in overweight and obese subjects. Interestingly, fasting insulin or HOMA-IR were not predictive factors in the development of IFG. ${ }^{24}$

There are some inherent limitations in this study. We were unable to validate our preliminary findings with direct quantification of IAAT and insulin sensitivity measured by clamps and we did not confirm in a lean control group. Our cohort was heterogeneous in age, obesity status, and sexual maturation. However, we assessed the changes in metabolic risk according to median age (14.5 years) and found no significant differences in the metabolic response to intervention. There were no significant differences in metabolic improvements according to those above or below the median waist circumference $(96 \mathrm{~cm})$. Furthermore, we were not able to consider the effect of differences in sexual maturity, although dyslipidemia only worsens at the onset of puberty and remains stable during all other pubertal stages. ${ }^{25}$

In summary, fasting TG within the HyperTG waist phenotype is an independent predictor and accounts for the majority of the variance of the metabolic triad. Weight lossassociated improvements in fasting TG concentration (not waist circumference) predicted the cumulative change in the MTS (fasting insulin, ApoB, LDL peak particle density) that is characteristic of IAAT, insulin resistance, and metabolic obesity. Therefore, fasting TG concentrations may be a marker of metabolically abnormal obesity and a surrogate of underlying insulin resistance within the metabolic syndrome definitions. Furthermore, the changes in TG may characterize the metabolic improvements in response to weight loss in clinically obese youth.

\section{Acknowledgements}

The authors would like to thank the children and adolescents who participated in the study.

\section{Author Disclosure Statement}

No competing financial interests exist. J.P.H. collected all data. J.P.H. and S.C. conducted all statistical analyses and wrote the manuscript. R.F.K., P.P., A.S., J.H.B., and P.G. reviewed/edited the manuscript.

\section{References}

1. Johnson WD, Kroon JJ, Greenway FL, et al. Prevalence of risk factors for metabolic syndrome in adolescents: National Health and Nutrition Examination Survey (NHANES), 2001-2006. Arch Pediatr Adolesc Med 2009;163:371-377.

2. Taksali SE, Caprio S, Dziura J, et al. High visceral and low abdominal subcutaneous fat stores in the obese adolescent: A determinant of an adverse metabolic phenotype. Diabetes 2008;57:367-371.
3. Weiss R, Dufour S, Taksali SE, et al. Prediabetes in obese youth: A syndrome of impaired glucose tolerance, severe insulin resistance, and altered myocellular and abdominal fat partitioning. Lancet 2003;362:951-957.

4. Lamarche B, Tchernof A, Mauriege P, et al. Fasting insulin and apolipoprotein $B$ levels and low-density lipoprotein particle size as risk factors for ischemic heart disease. JAMA 1998;279:1955-61.

5. Lemieux I, Pascot A, Couillard C, et al. Hypertriglyceridemic waist: A marker of the atherogenic metabolic triad (hyperinsulinemia; hyperapolipoprotein $\mathrm{B}$; small, dense LDL) in men? Circulation 2000;102:179-184.

6. Gately PJ, Cooke CB, Barth JH, et al. Children's residential weight-loss programs can work: A prospective cohort study of short-term outcomes for overweight and obese children. Pediatrics 2005;116:73-77.

7. Cole TJ, Bellizzi MC, Flegal KM, et al. Establishing a standard definition for child overweight and obesity worldwide: International survey. Br Med J 2000;320:1240-1243.

8. Schofield WN. Predicting basal metabolic rate, new standards and review of previous work. Hum Nutr Clin Nutr 1985;39(Suppl 1):5-41.

9. Duckworth LC, Gately PJ, Radley D, et al. RCT of a highprotein diet on hunger motivation and weight-loss in obese children: An extension and replication. Obesity (Silver Spring) 2009;17:1808-1810.

10. Walker LL, Gately PJ, Bewick BM, et al. Children's weightloss camps: Psychological benefit or jeopardy? Int J Obes Relat Metab Disord 2003;27:748-754.

11. Jebb SA, Cole TJ, Doman D, et al. Evaluation of the novel Tanita body-fat analyser to measure body composition by comparison with a four-compartment model. $\mathrm{Br} \mathrm{J}$ Nutr 2000;83:115-122.

12. Davies IG, Graham JM, Griffin BA. Rapid separation of LDL subclasses by iodixanol gradient ultracentrifugation. Clin Chem 2003;49:1865-1872.

13. de Ferranti SD, Gauvreau K, Ludwig DS, et al. Prevalence of the metabolic syndrome in American adolescents: Findings from the Third National Health and Nutrition Examination Survey. Circulation 2004;110:2494-2497.

14. Zhu S, Wang $Z$, Heshka $S$, et al. Waist circumference and obesity-associated risk factors among whites in the third National Health and Nutrition Examination Survey: Clinical action thresholds. Am J Clin Nutr 2002;76:743-749.

15. Eisenmann JC, Laurson KR, DuBose KD, et al. Construct validity of a continuous metabolic syndrome score in children. Diabetol Metab Syndr 2010;2:8.

16. Monzavi R, Dreimane D, Geffner ME, et al. Improvement in risk factors for metabolic syndrome and insulin resistance in overweight youth who are treated with lifestyle intervention. Pediatrics 2006;117:e1111-e1118.

17. Savoye $M$, Shaw M, Dziura J, et al. Effects of a weight management program on body composition and metabolic parameters in overweight children: A randomized controlled trial. JAMA 2007;297:2697-2704.

18. Miller $M$, Stone NJ, Ballantyne $C$, et al. Triglycerides and cardiovascular disease: A scientific statement from the American Heart Association. Circulation 2011;123:2292-2333.

19. Primeau V, Coderre L, Karelis AD, et al. Characterizing the profile of obese patients who are metabolically healthy. Int J Obes (Lond) 2011;35:971-981.

20. Cali AM, Zern TL, Taksali SE, et al. Intrahepatic fat accumulation and alterations in lipoprotein composition in obese adolescents: A perfect proatherogenic state. Diabetes Care 2007;30:3093-3098. 
21. McLaughlin T, Abbasi F, Cheal K, et al. Use of metabolic markers to identify overweight individuals who are insulin resistant. Ann Internal Med 2003;139:802-809.

22. Hannon TS, Bacha F, Lee SJ, et al. Use of markers of dyslipidemia to identify overweight youth with insulin resistance. Pediatr Diabetes 2006;7:260-266.

23. Marotta T, Russo BF, Ferrara LA. Triglyceride-to-HDLcholesterol ratio and metabolic syndrome as contributors to cardiovascular risk in overweight patients. Obesity (Silver Spring) 2010;18:1608-1813.

24. Shand BI, Scott RS, Lewis JG, et al. Comparison of indices of insulin resistance with metabolic syndrome classifications to predict the development of impaired fasting glucose in overweight and obese subjects: A 3-year prospective study. Int J Obes (Lond) 2009;33:1274-1279.
25. Pinhas-Hamiel O, Lerner-Geva L, Copperman NM, et al. Lipid and insulin levels in obese children: changes with age and puberty. Obesity (Silver Spring) 2007;15:2825-2831.

Address correspondence to: Dr. James P Hobkirk Department of Academic Endocrinology, Diabetes and Metabolism Hull York Medical School University of Hull Hull HU6 TRX United Kingdom

E-mail: james.hobkirk@hyms.ac.uk 